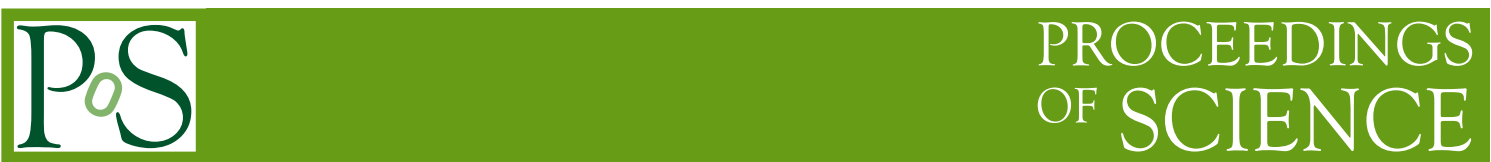

\title{
Automated lattice perturbation theory applied to HQET
}

\section{Dirk Hesse*}

NIC, DESY, Platanenallee 6, 15738 Zeuthen, Germany

Current address: Universitá degli studi di Parma, Viale G.P. Usberti 7/a, 43100 Parma, Italy

E-mail: dirk.hessedfis.unipr.it

\section{Rainer Sommer}

NIC, DESY, Platanenallee 6, 15738 Zeuthen, Germany

\section{Georg von Hippel}

Universität Mainz, Institut für Kernphysik, Becherweg 45, 55099 Mainz, Germany

We present the pastor software package for automated lattice perturbation theory calculations in the Schrödinger functional scheme. We discuss automatic diagram and vertex generation and present an example calculation involving correlation functions using both the heavy quark effective theory (HQET) action including $1 / m$ corrections and the $O(a)$-improved Wilson action.

XXIX International Symposium on Lattice Field Theory

July $10-162011$

Squaw Valley, Lake Tahoe, California

\footnotetext{
* Speaker.
} 


\section{Introduction}

In lattice gauge theories, a number of different lattice prescriptions of the continuum action can be used. Different lattice actions often provide specific advantages and disadvantages, such that in practice various actions are in use. Each of these yields different Feynman rules, such that a given lattice perturbation theory calculation in principle has to be repeated if one chooses to work with another action. It is thus beneficial if one uses an automated method to derive the Feynman rules. Such a method is discussed in [1,2] and a variant of it will be employed in this work.

In the Schrödinger functional scheme [9,7], two more issues arise. Firstly, one has to take the induced background field into account when constructing the Feynman rules. This can be accomplished with a modified version of the aforementioned method, as discussed in [8] for the gauge sector. Secondly, the Schrödinger functional boundary conditions increase the number of Feynman diagrams contributing to a process already at one loop order.

One of the authors (D.H.) developed the pastor software package, which addresses all of these issues. For a rather general class of correlation functions and actions in the Schrödinger functional, pastor automatically calculates the perturbative expansion up to order $g_{0}^{2}$ in the bare coupling, including automatic generation of vertices and diagrams.

In the following, we will describe the methods used by past or to generate diagrams and vertices and present an example calculation, involving correlation functions in heavy quark effective theory (HQET).

\section{Background}

We work in the Schrödinger functional scheme, using the same notation as in [6] with a more general fermion action $S_{F}[\bar{\psi}, \psi]$. The quark fields $\psi, \bar{\psi}$ obey the boundary conditions

$$
\begin{array}{ll}
\left.P_{+} \psi(x)\right|_{x_{0}=0}=\rho(\mathbf{x}), & \left.P_{-} \psi(x)\right|_{x_{0}=T}=\rho^{\prime}(\mathbf{x}), \\
\left.\bar{\psi}(x) P_{-}\right|_{x_{0}=0}=\bar{\rho}(\mathbf{x}), & \left.\bar{\psi}(x) P_{+}\right|_{x_{0}=T}=\bar{\rho}^{\prime}(\mathbf{x}) .
\end{array}
$$

The spatial boundary conditions are $L$-periodic up to a phase, $\psi(x+L \hat{k})=e^{i \theta_{k}} \psi(x)$. The Dirac operator is defined through the action,

$$
\frac{\delta}{\delta \bar{\psi}(x)} S_{F}=(D+m) \psi(x), \quad 0<x_{0}<T,
$$

and we define the kernels $K, K^{\prime}, \tilde{K}, \tilde{K}^{\prime}$ through the action at the boundary,

$$
\begin{gathered}
-\frac{\delta}{\delta \rho(\mathbf{x})} S_{F}=-\sum_{y} \bar{\psi}(y) K(y, \mathbf{x}), \quad \frac{\delta}{\delta \bar{\rho}(\mathbf{x})} S_{F}=-\sum_{y} \tilde{K}(\mathbf{x}, y) \psi(y) \\
-\frac{\delta}{\delta \rho^{\prime}(\mathbf{x})} S_{F}=-\sum_{y} \bar{\psi}(y) K^{\prime}(y, \mathbf{x}), \quad \frac{\delta}{\delta \bar{\rho}^{\prime}(\mathbf{x})} S_{F}=-\sum_{y} \tilde{K}^{\prime}(\mathbf{x}, y) \psi(y),
\end{gathered}
$$

where we now understand $0 \leq y_{0} \leq T$ and the kernels obey the boundary conditions

$$
\left.P_{+} K(y, \mathbf{x})\right|_{y_{0}=0}=\left.\tilde{K}(\mathbf{x}, y) P_{-}\right|_{y_{0}=0}=\left.P_{-} K^{\prime}(y, \mathbf{x})\right|_{y_{0}=T}=\left.\tilde{K}(\mathbf{x}, y) P_{+}\right|_{y_{0}=T}=0 .
$$


The $S U(N)$ lattice gauge fields $U_{\mu}(x)=U_{-\mu}^{-1}(x+a \hat{\mu})$ in the Schrödinger functional are $L$-periodic in the spatial directions and obey the boundary conditions

$$
\left.U_{k}(x)\right|_{x_{0}=0}=W_{k}(\mathbf{x}),\left.\quad U_{k}(x)\right|_{x_{0}=T}=W_{k}^{\prime}(\mathbf{x}) .
$$

The boundary links $U_{0}(x)$ are unrestricted. The relevant degrees of freedom for perturbation theory are the gauge fields in an infinitesimal neighborhood of the classical solution $V_{\mu}$ minimizing the action under the boundary conditions (2.5). These fields may be parametrized as

$$
U_{\mu}(x)=\exp \left\{g_{0} q_{\mu}(x)\right\} V_{\mu}(x) .
$$

\subsection{Automatic Generation of Feynman Diagrams}

Following the basic steps of [6] using the general form of the fermion action defined above, one can split up the expectation value of any observable $\mathscr{O}$ given in terms of the quark fields $\bar{\psi}, \psi$ and the gluon fields $U_{\mu}(x)$ in a gauge and fermion average $\langle\mathscr{O}\rangle=\left\langle[\mathscr{O}]_{F}\right\rangle_{G}$, for details c.f. [6]. The fermion average may be calculated using the generating functional

$$
\mathscr{Z}^{F}\left[\bar{\rho}, \bar{\rho}^{\prime}, \bar{\eta}, \rho, \rho^{\prime}, \eta\right]=\int \mathscr{D}[\bar{\psi}, \psi] \exp \left\{-S_{F}[\bar{\psi}, \psi]+\bar{\psi}(x) \eta(x)+\bar{\eta}(x) \psi(x)\right\},
$$

with the source fields $\bar{\eta}(x), \eta(x), 0<x_{0}<T$. Useful observables may also contain the boundary fields [10]

$$
\zeta(\mathbf{x})=\frac{\delta}{\delta \bar{\rho}(\mathbf{x})}, \quad \bar{\zeta}(\mathbf{x})=-\frac{\delta}{\delta \rho(\mathbf{x})}, \quad \zeta^{\prime}(\mathbf{x})=\frac{\delta}{\delta \bar{\rho}^{\prime}(\mathbf{x})}, \quad \bar{\zeta}^{\prime}(\mathbf{x})=-\frac{\delta}{\delta \rho^{\prime}(\mathbf{x})}
$$

After these derivatives are applied to the Boltzmann factor in (2.7), the fields $\rho, \rho^{\prime}, \bar{\rho}, \bar{\rho}^{\prime}$ are set to zero. Defining the Green's function $S$ of the Dirac operator for a given gauge field,

$$
(D+m) S(x, y)=\delta_{x y}, \quad 0<x_{0}<T,\left.\quad P_{+} S(x, y)\right|_{x_{0}=0}=\left.P_{-} S(x, y)\right|_{x_{0}=T}=0,
$$

one may construct the general classical solution $\psi_{\mathrm{cl}}$ (and its adjoint $\bar{\psi}_{\mathrm{cl}}$ accordingly)

$$
\psi_{\mathrm{cl}}(x)=\sum_{y, \mathbf{Z}} S(x, y)\left\{K(y, \mathbf{z}) \rho(\mathbf{z})+K^{\prime}(y, \mathbf{z}) \rho^{\prime}(\mathbf{z})\right\}, \quad 0<x_{0}<T,
$$

such that $(D+m) \psi_{\mathrm{cl}}(x)=0$ for $0<x_{0}<T$ with the boundary conditions

$$
\left.P_{+} \psi_{\mathrm{cl}}(x)\right|_{x_{0}=0}=\rho(\mathbf{x}),\left.\quad P_{-} \psi_{\mathrm{cl}}(x)\right|_{x_{0}=T}=\rho^{\prime}(\mathbf{y}) .
$$

Making use of $\psi_{\mathrm{cl}}$, the generating functional (2.7) may be expressed in a simple way,

$$
\begin{aligned}
\ln \mathscr{Z}^{F}= & \ln \mathscr{Z}^{F}[0]+\sum_{x, y} \bar{\eta}(x) S(x, y) \eta(y)+\sum_{x}\left\{\bar{\eta}(x) \psi_{\mathrm{cl}}(x)+\bar{\psi}_{\mathrm{cl}}(x) \eta(x)\right\} \\
& +\sum_{\mathbf{x}, y}\left\{\bar{\rho}(\mathbf{x}) \tilde{K}(\mathbf{x}, y)+\bar{\rho}^{\prime}(\mathbf{x}) \tilde{K}^{\prime}(\mathbf{x}, y)\right\} \psi_{\mathrm{cl}}(y) .
\end{aligned}
$$

With (2.12), one may calculate all basic Wick contractions by differentiation with respect to the boundary values and source fields. All of those contractions can be written in terms of the propagator $S$ and the kernels $K, K^{\prime}, \tilde{K}, \tilde{K}^{\prime}$. For example, consider

$$
[\psi(x) \bar{\zeta}(\mathbf{z})]_{F}=\left.\frac{1}{\mathscr{Z}}\left(\frac{\delta}{\delta \bar{\eta}(x)}\right)\left(-\frac{\delta}{\delta \rho(\mathbf{z})}\right) \mathscr{Z}\right|_{\bar{\rho}=\ldots=\eta=0}=\sum_{y} S(x, y) K(y, \mathbf{z}) P_{+} .
$$


We may now expand $S$ in powers of the bare coupling $g_{0}$ by solving

$$
\left[D^{(0)}+m+\sum_{i=1}^{\infty} g_{0}^{i} D^{(i)}\right] \sum_{j=0}^{\infty} g_{0}^{j} S^{(j)}(x, y)=\delta_{x y}
$$

order by order in $g_{0}$ [6]. This involves the inversion ${ }^{1}$ of $\left(D^{(0)}+m\right)$ to obtain $S^{(0)}$ and then the explicit form of $D^{(i)}$ is needed to construct the higher order terms, e.g.

$$
S^{(1)}(x, z)=-\sum_{y} S^{(0)}(x, y) D^{(1)} S^{(0)}(y, z)
$$

In the following section, we will present a method to calculate the vertices $D^{(i)}$ for a general action. This method is then also used to construct the $g_{0}$-expansion of $K, K^{\prime}, \tilde{K}$ and $\tilde{K}^{\prime}$, such that we get e.g. for (2.13)

$$
[\psi(x) \bar{\zeta}(\mathbf{z})]_{F}=\sum_{y}\left(\sum_{i} g_{0}^{i} S^{(i)}(x, y)\right)\left(\sum_{j} g_{0}^{j} K^{(j)}(y, \mathbf{z})\right) P_{+} .
$$

The pastor package consists of a frontend written in Python, whose input is an observable, consisting of a number of traces $T_{i},{ }^{2}$ where each trace is a product of a number of propagators $S_{j}$, boundary kernels $K_{k}, K_{l}^{\prime}, \ldots$, and insertions $\kappa_{m}$ coming from terms of the form $\sum_{x, y} \bar{\psi}(x) \kappa(x, y) \psi(x)$ in the observable. It then performs the $g_{0}$-expansion symbolically in the spirit of (2.16), aborting at $O\left(g_{0}^{2}\right)$. This yields expressions with two free gluons, $q_{\mu}^{a}(x), q_{v}^{b}(y) .{ }^{3}$ The last step is to calculate the gluon average, which is accomplished at order $g_{0}^{2}$ by contracting the free gluons into a gluon propagator $D_{\mu \nu}^{a b}(x, y)$. After gauge fixing, the latter is calculated in the same fashion as the fermion propagator. The backend is a $\mathrm{C}++$ library that constructs vertices from any gauge or fermion action, which can be passed to the code at run-time in a symbolic form, as will be explained in detail in the next section. The frontend will produce $\mathrm{C}++$ programs making use of the backend to calculate all diagrams up to and including order $g_{0}^{2}$, with all required counter-terms. The package uses spatial translational invariance, evaluating the Feynman diagrams in a $x_{0}, \mathbf{p}$ representation.

\subsection{Automatic Generation of Vertices}

The automatic generation of vertices in the Schrödinger functional for the gluon sector has been discussed in depth in [8]. We will focus here on the fermion sector. A typical bilinear fermion action $S_{F}$ may be written as a sum over parallel transporters $\mathscr{U}_{i}\left(x^{i}, y^{i}\right)$ with weights $c_{i}$ and spin matrices $\Gamma_{i}$ (which can be Pauli or Dirac matrices, as well as scalars),

$$
S_{F}[\bar{\psi}, \psi, U]=\sum_{i} c_{i} \bar{\psi}\left(x^{i}\right) \Gamma_{i} \mathscr{U}_{i}\left(x^{i}, y^{i}\right) \psi\left(y^{i}\right), \quad \mathscr{U}_{i}\left(x^{i}, y^{i}\right)=U_{n_{i}}^{i} \ldots U_{2}^{i} U_{1}^{i} .
$$

We then express the $D^{(k)}$ in the expansion of the Dirac operator (2.14) in terms of the $q_{\mu}$ field,

$$
D^{(k)}=\frac{1}{k !} \sum_{x_{1}, \mu_{1}, a_{1}} \ldots \sum_{x_{k}, \mu_{k}, a_{k}} q_{\mu_{1}}^{a_{1}}\left(x_{1}\right) \ldots q_{\mu_{k}}^{a_{k}}\left(x_{k}\right) \sum_{y, z, b, c} \bar{\psi}_{b}(y) \mathscr{V}_{\mu_{1}, \ldots, \mu_{k}}^{a_{1}, \ldots, a_{k}, b, c}\left(x_{1}, \ldots, x_{k} ; y, z\right) \psi_{c}(z) .
$$

\footnotetext{
${ }^{1}$ For many actions, the free propagator is known. To stay independent of specific formulations of the lattice theory, pastor includes a number of inverters to calculate the free propagator numerically.

${ }^{2} \mathrm{An}$ arbitrary number of traces is at the time of writing only supported for a trivial background field $V_{\mu}(x) \equiv 1$.

${ }^{3}$ With a non-trivial background field, the situation is more complicated, c.f. [12].
} 
The vertex functions $\mathscr{V}$ may then be written as symmetrized products of reduced vertex functions $Y_{\mu_{1}, \ldots, \mu_{k}}\left(x_{1}, \ldots, x_{k} ; y, z\right)$ and color factors $C$ [2], where the reduced vertex functions are calculated by summing over contributions from each of the terms in (2.17). The part coming from an individual parallel transporter $\mathscr{U}_{i}$ can in turn be expressed as a function of the positions $x_{j}^{i}$ and the Lorentz indices $\mu_{j}^{i}$. The basic strategy is to start from the expansion fo a single link (2.6) and build up the expansion of any parallel transporter iteratively by multiplication.

For a trivial background field $V_{\mu} \equiv 1$, the color factor is simply given by a product of generators $T^{a}$ of $S U(N)$,

$$
C^{a_{1}, \ldots, a_{k}, b, c}=\left(T^{a_{1}} \ldots T^{a_{k}}\right)_{b c} .
$$

For an Abelian background field as defined in [7], the color factor assumes a more complicated form [8],

$$
C^{a_{1}, \ldots, a_{k}, b, c}(t, A, B, C, D)=\left[I^{a_{1}} \ldots I^{a_{k}} V(t)^{A} e^{i \mathscr{E} B} \exp \left\{i / 2 \sum_{j=1}^{k}\left(C_{j} \phi_{a_{j}}^{\prime}+D_{j} \phi_{a_{j}}(t)\right)\right\}\right]_{b c},
$$

where the scalars $A, B$ and the $k$-vectors $C, D$ may again be calculated iteratively for each of the parallel transporters in (2.17) starting from a single link. $\mathscr{E}, \phi^{\prime}$, and $\phi(t)$ are depending on the background field and the $I^{a}$ are another set of generators of $S U(N)$. Both (2.19) and (2.20) can be chosen to be used in pastor, such that one can switch between Abelian and trivial background field easily. Given a number of parallel transporters $\mathscr{U}_{i}$ e.g. as lists of directions $\mu_{j}^{i}$, the corresponding weights $c_{i}$, and spin matrices $\Gamma_{i}$ as in (2.17) as input, the $\mathrm{C}++$ backend of pastor will calculate the vertices $\mathscr{V}$ to any order in $g_{0}$. The gluon action is treated in a similar way [8]. The pastor backend may hence be used for perturbative calculations in the Schrödinger functional independently of the frontend, and therefore in principle also beyond the $g_{0}^{2}$ terms.

\section{Application to HQET}

Any expectation value $\langle\mathscr{O}\rangle$ in HQET may be expanded in the following way [14],

$$
\langle\mathscr{O}\rangle=\langle\mathscr{O}\rangle_{\text {stat }}+\omega_{\text {spin }}\langle\mathscr{O}\rangle_{\text {spin }}+\omega_{\text {kin }}\langle\mathscr{O}\rangle_{\text {kin }}, \quad\langle\mathscr{O}\rangle_{\text {kin }}=\sum_{y}\left\langle\mathscr{O} \mathscr{O}_{\text {kin }}(y)\right\rangle_{\text {stat }}, \quad \text { etc. }
$$

where $\langle\cdot\rangle_{\text {stat }}$ is the path integral average using the static Lagrangian $\mathscr{L}_{\text {stat }}$ and $\mathscr{O}_{\text {kin }}, \mathscr{O}_{\text {spin }}$ contribute at $O(1 / \mathrm{m})$. In the first of a series of publications on HQET including $1 / \mathrm{m}$ effects, the ALPHA collaboration describes the non-perturbative determination of the required parameters $\omega_{\text {kin }}$ etc. [3]. The main idea of the method employed there may be summarized as follows. In a physically small volume with spatial extend $L_{1}$, where simulations in HQET and QCD are feasible, one matches the two theories by requiring the equality of a number of observables $\Phi_{i}$ in HQET at various lattice spacings with the continuum limit of their QCD counterparts. One then proceeds with a finite size scaling method to determine $\Phi_{i}$ in a larger volume $L_{2}$. This procedure involves a number of step scaling functions $\Sigma_{i j}$. To demonstrate the effectiveness of pastor, we calculated the one-loop cutoff effects of the functions $\Sigma_{33}$ and $\Sigma_{44}$ [3],

$$
\begin{gathered}
\Sigma_{33}\left(L_{1}\right)=R_{\delta A}\left(L_{2}\right) / R_{\delta A}\left(L_{1}\right), \quad \Sigma_{44}\left(L_{1}\right)=R_{1}^{\mathrm{kin}}\left(L_{2}\right) / R_{1}^{\mathrm{kin}}\left(L_{1}\right), \\
R_{\delta A}(L)=\left(\frac{f_{\delta A}^{\text {stat }}\left(\theta, \frac{T}{2}\right)}{f_{A}^{\text {stat }}\left(\theta, \frac{T}{2}\right)}-\frac{f_{\delta A}^{\mathrm{stat}}\left(\theta^{\prime}, \frac{T}{2}\right)}{f_{A}^{\text {stat }}\left(\theta^{\prime}, \frac{T}{2}\right)}\right)_{T=L}, \quad R_{1}^{\mathrm{kin}}(L)=\left(\frac{f_{1}^{\mathrm{kin}}(\theta)}{f_{1}^{\text {stat }}(\theta)}-\frac{f_{1}^{\mathrm{kin}}\left(\theta^{\prime}\right)}{f_{1}^{\text {stat }}\left(\theta^{\prime}\right)}\right)_{T=\frac{L}{2}} .
\end{gathered}
$$


The correlation functions in these expressions are part of the expansion (3.1) of the heavy-light correlation functions

$$
f_{A}\left(\theta, x_{0}\right)=-\frac{a^{6}}{2} \sum_{\mathbf{y}, \mathbf{z}}\left\langle\left(A_{I}\right)_{0}(x) \bar{\zeta}_{h}(\mathbf{y}) \gamma_{k} \zeta_{l}(\mathbf{z})\right\rangle, \quad f_{1}(\theta)=-\frac{a^{12}}{2 L^{6}} \sum_{\mathbf{u}, \mathbf{v}, \mathbf{y}, \mathbf{z}}\left\langle\bar{\zeta}_{l}^{\prime}(\mathbf{u}) \gamma_{5} \zeta_{h}^{\prime}(\mathbf{v}) \bar{\zeta}_{h}(\mathbf{y}) \gamma_{5} \zeta_{l}(\mathbf{z})\right\rangle .
$$

$f_{\delta A}$ is defined similar to $f_{A}$, making the replacement $\left(A_{I}\right)_{0} \rightarrow \delta A_{0}$. The insertion $\delta A$ stems from the $O(1 / \mathrm{m})$ correction to the static-light axial current. For the discretization of the light flavor, we choose $O(a)$ improved Wilson fermions, the gauge action used here is the Wilson plaquette action.

\subsection{Perturbative Improvement of Observables}

Perturbative improvement [5] has been shown to be an effective method to speed up the approach to the continuum limit of a number of observables. The aim is to remove at a given order of perturbation theory all $O\left((a / L)^{n} \log (L / a)^{m}\right)$ cutoff effects. One defines the improved observable

$$
\mathscr{O}_{\mathrm{I}}(a / L)=\frac{\mathscr{O}(a / L)}{1+\delta(a / L)}, \quad \delta(a / L)=\frac{\mathscr{O}(a / L)-\mathscr{O}(0)}{\mathscr{O}(0)}=\delta^{(0)}(a / L)+g_{0}^{2} \delta^{(1)}(a / L)+\ldots
$$

The tree level improvements $\delta_{i j}^{(0)}$ have been calculated for the step-scaling functions $\Sigma_{i j}$ in [3]. The one loop improvements $\delta_{33}^{(1)}$ and $\delta_{44}^{(1)}$ for $\Sigma_{33}$ and $\Sigma_{44}$ are presented in figure 1, together with the tree level improved Monte Carlo data from [13]. One should keep in mind that our investigation uses a different discretization (Eichten-Hill, [11]) than the ones (HYP1/2) employed in [3]. However, the sizes of the cutoff effects are qualitatively similar. Perturbation theory provides an interesting hint for non-perturbative computations: the cutoff effects are minimal for $\theta=0.5, \theta^{\prime}=1.0$.

\subsection{Extrapolation of perturbative data.}

To perform the necessary continuum extrapolations for (3.4), we used the method of successive fits proposed in [12]. To have good control over cutoff effects, we performed calculations with $L / a=4,6, \ldots, 48$ for all observables. pastor provides an option to perform all computations in long double precision to estimate the roundoff-errors, wich turn out to be negligible in our case.

\section{Acknowledgements}

We thank S. Takeda, U. Wolff, and H. Simma for helpful discussions. Our computations were performed on the DESY computer farm in Zeuthen. We thank the autors of [13] and in particual N. Garron for providing the Monte Carlo data for fig. 1. This work has been supported by the DFG Sonderforschungsbereich TR9 Computergestützte Theoretische Teilchenphysik and the Research Executive Agency (REA) of the European Union under Grant Agreement number PITN-GA-2009238353 (ITN STRONGnet).

\section{References}

[1] M. Lüscher, P. Weisz, Nucl. Phys. B266, 309 (1986).

[2] A. Hart, G. M. von Hippel, R. R. Horgan, E. H. Müller, Comput. Phys. Commun. 180, 2698-2716 (2009). [arXiv:0904.0375 [hep-lat]]. 




Figure 1: One loop cutoff effects of $\Sigma_{33}$ and $\Sigma_{44}$ (Eichten-Hill action), together with the tree level improved Monte Carlo results ( $N_{f}=2, g_{0} \sim 1$, HYP2 action) for comparison. The numerically most challenging diagram took 3.5 days to be evaluated for $L / a=48$ on a single $2 \mathrm{GHz}$ CPU core.

[3] B. Blossier, M. della Morte, N. Garron, R. Sommer, JHEP 1006, 002 (2010). [arXiv:1001.4783 [hep-lat]].

[4] M. Della Morte, N. Garron, M. Papinutto, R. Sommer, JHEP 0701, 007 (2007). [hep-ph/0609294].

[5] G. de Divitiis et al. [ ALPHA Collaboration ], Nucl. Phys. B437, 447-470 (1995). [hep-lat/9411017].

[6] Lüscher, M. and Weisz, P. Nucl. Phys. B479 429-458 (1996). [hep-lat/9606016]

[7] M. Lüscher, R. Narayanan, P. Weisz, U. Wolff, Nucl. Phys. B384, 168-228 (1992). [hep-lat/9207009].

[8] S. Takeda, Nucl. Phys. B811, 36-65 (2009). [arXiv:0808.3065 [hep-lat]].

[9] S. Sint, Nucl. Phys. B421, 135-158 (1994). [hep-lat/9312079].

[10] M. Lüscher, S. Sint, R. Sommer, P. Weisz, Nucl. Phys. B478, 365-400 (1996). [hep-lat/9605038].

[11] E. Eichten, B. R. Hill, Phys. Lett. B243, 427-431 (1990).

[12] A. Bode et al. [ ALPHA Collaboration ], Nucl. Phys. B576, 517-539 (2000). [hep-lat/9911018].

[13] B. Blossier et al. [ ALPHA Collaboration ], PoS LATTICE2010, 308 (2010). [arXiv:1012.1357 [hep-lat]].

[14] J. Heitger et al. [ ALPHA Collaboration ], JHEP 0402, 022 (2004). [hep-lat/0310035]. 